Review Article

\section{The role of robotic segmentectomy for non-small cell lung cancer}

\author{
Anil Gokce ${ }^{1 *}$, Yucel Akkas² and Bulent Kocer ${ }^{3}$ \\ ${ }^{1}$ Thoracic Surgeon, University of Health Sciences, Ankara City Hospital, Department of Thoracic \\ Surgery, Bilkent, Ankara, Turkey \\ ${ }^{2}$ Associate Professor, University of Health Sciences, Ankara City Hospital, Department of Thoracic \\ Surgery, Bilkent, Ankara, Turkey \\ ${ }^{3}$ Professor, University of Health Sciences, Ankara City Hospital, Department of Thoracic Surgery, \\ Bilkent, Ankara, Turkey
}

\section{Abstract}

Segmentectomy may be applied to all segments; superior segmentectomies (lower lobe superior segments for both lungs), lingulectomies (two segments forming lingulas of upper left lobe) and basal segmentectomies (segments other than superior segment for both lungs). In lung segment resections; segmentectomy has an equivalent morbidity, recurrence and survival rate compared to lobectomy, in patients with stage I lung cancer, tumors smaller than $2 \mathrm{~cm}$ and within the segmental anatomical limits. Segmentectomy also contributes more to preserving lung function and exercise capacity than lobectomy. In tumor resection; especially in patients with advanced age, insufficient performance or insufficient cardiopulmonary reserve, $2 \mathrm{~cm}$ in diameter and acceptable segmental margins may be provided.

Limited long-term results show oncological results of robotic approach similar to open and VATS approaches. Robotic approach facilitates surgery with more intuitive movements, greater flexibility and high definition, three-dimensional vision. However, high cost and lack of touch sense are main disadvantages of robotic surgery. New studies are needed to assess quality of life, morbidity, oncological results and cost effectiveness. However, considering development of technology in our age and fact that many surgical robot brands will be released in the near future, it is predicted that disadvantages of robotic surgery will be minimized in the near future.

This article reviews experience of segmentectomy in non-small cell lung cancer and discusses benefits and limitations of robotic segmentectomy.
More Information

*Address for Correspondence: Anil Gokce, MD, Thoracic Surgeon, University of Health Sciences, Ankara City Hospital, Department of Thoracic Surgery, Bilkent, Ankara, Turkey, Tel: +90 31255260 00; +90 50525924 15; Email: anil66gokce@hotmail.com

Submitted: 02 September 2020

Approved: 05 October 2020

Published: 06 October 2020

How to cite this article: Gokce A, Akkas Y, Kocer $B$. The role of robotic segmentectomy for non-small cell lung cancer. Arch Surg Clin Res. 2020; 4: 042-046.

DOI: 10.29328/journal.ascr.1001052

Copyright: ( 2020 Gokce A, et al. This is an open access article distributed under the Creative Commons Attribution License, which permits unrestricted use, distribution, and reproduction in any medium, provided the original work is properly cited.

Keywords: Segmentectomy; Robotic segmentectomy; Robot assisted thoracic surgery; Minimal invasive surgery

Check for updates

OPEN ACCESS

\section{Introduction}

Sublobar resections includes wedge resection and anatomic segment resections. However, wedge resections are not applied in the treatment of lung cancer and are mostly used for benign conditions. Sublobar resections referred to anatomic segment resections in this article. Segmentectomy may be applied to all segments; superior segmentectomies (lower lobe superior segments for both lungs), lingulectomies (two segments forming lingulas of upper left lobe), basal segmentectomies (segments other than superior segment for both lungs) and left upper lobe apical trisegmentectomy (lingula sparing LUL). In lung, there are a total of 10 segments in right; 3 in upper lobe, 2 in middle lobe, 5 in lower lobe and a total of 8 segments in left; 4 in upper lobe and 4 in lower lobe. Segmentectomy is smallest anatomical lung resections that may be operated. Dissection should be made into parenchyma to reach anatomical structures belonging to segment. General procedure is completion resection with stripping after segment artery and bronchus dissected and ligated. In practice, after dissection of artery and bronchus, lung is ventilated and unventilated area is resected with staples [1].

\section{Segmentectomy in non-small cell lung cancer treatment}

Until the mid-1900s, all clinical evidence for sublobar resections consisted of retrospective case series. In 1973, a series of 69 patients that underwent segmentectomy due to bronchogenic carcinoma showed 5-year survival was 56\% [2]. In 1990, Read, et al. compared T1N0 non-small cell lung cancer (NSCLC) patients who underwent 131 lobectomy and 113 sublobar resection (including 107 segmentectomy). They did not find a difference in cancer-specific survival between groups and the estimated rate in the sublobar group was as high as $92 \%$. Despite the promising results, it was difficult to 
deduce a reliable conclusion that sublobar resections were superior or at least equal to lobectomy due to deficiencies in studies [3]. For example, some early studies did not limit resection to stage I disease; in some studies, acceptance and rejection criteria for sublobar resection were variable and no cancer-specific survival was considered in any study. As a result, clinical evidence was insufficient to emphasize the role of sublobar resections. In 1995, Ginsberg, et al. published a single randomized study that compared sublobar resection and lobectomy in T1N0 NSCLC. In this study, 122 patients (including 82 segmentectomy) who underwent sublobar resection were compared with 125 patients who underwent lobectomy. The results showed that annual mortality rate was $30 \%$ higher in the sublobar group as close to statistical significance. However, the locoregional recurrence rate was subjectively $300 \%$ higher in the sublobar group than in the lobectomy group, and wedge resection results were worse than segmentectomy [4]. This result confirmed the worries that sublobar resection was insufficient in oncological treatment. This publication has often been used to criticize the use of sublobar resections, and over the following years the use of this approach in lung cancer varied among surgeons. In 2005, Nakamura, et al. published the first main meta-analysis of sublobar resection results. For this metaanalysis, a total of 14 studies were selected including 12 retrospective case series. Sublobar resection was applied to 903 patients and conventional lobectomy to 1887 patients. As a result, 3 studies concluded that lobectomy offers higher rate survival, while the other 13 did not find any difference [5]. Consequently, published data showed that sublobar resection is comparable to lobectomy in stage I lung cancer. De Zoysa, et al. conducted a systematic literature review. The authors discussed 16 studies about sublobar resection, including 8 studies published after the 2005 meta-analysis. The authors noticed that 3 studies showed a decreased survival in sublobar resection patients. However, further analysis showed that sublobar resection patients were older and lymph node sampling was limited. After adjusting these variables, the authors could not find a significant difference in survival. And the authors noticed that 6 studies showed that tumor size was an important factor. In these studies, it was presented survival of sublobar resection and lobectomy in tumors up to two centimeters in diameter and lobectomy was appeared superior in tumors larger than this size. 3 studies showed increased locoregional recurrence in sublobar resection. On the other hand, this 3 studies showed that after lung cancer surgery, sublobar resection significantly reduced morbidity, a decrease in complication rate, a decrease in hospital stay, and a more preserved lung function [6]. However, in both of the meta-analysis and systematic review, the authors suggested that much attention be paid to interpretation of the results. Since this time, many studies have confirmed that sublobar resection further preserves lung function. Harada, et al. showed that, segmentectomy FEV1 and FVC rates were significantly better than lobectomy [7]. In addition to maintaining lung function, general morbidity may be reduced by sublobar resection. This advantage may have important consequences, especially for older patients who cannot apply conventional lobectomy. Kilic, et al. stated that segmentectomy may reduce after surgery major complication rate from $25.5 \%$ to $11.5 \%$ in patients older than 75 years [8].

Donahue, et al. reported that local recurrence was 5\% and distant recurrence was $13 \%$ in stage IA patients with T1a lesions who underwent anatomic segmentectomy. The 5 -year recurrence-free survival of patients was $69 \%$. These improved results was the fact that some potential factors associated with better survival after sublobar resection were increasingly detectable. It was exemplified that tumor size $<2$ $\mathrm{cm}$, ensuring the adequacy of the surgical margin, performing segmentectomy instead of wedge resection and including nodal dissection in all types of sublobar resection [9]. In the study of Kates. et al. it was stated that stage I NSCLC patients who underwent 668 sublobar resections and 1402 lobectomies were identified using surveillance, epidemiology, and end results program (SEER) datas in the USA. In the study groups, it was determined that overall survival rates and lung cancer specific survival rates were not statistically different [10]. This result supports sublobar resection in smaller tumors.

Another important factor in success of sublobar resection is need for adequate lymph node sampling or dissection. In the study of Wolf, et al. it was reported that 238 patients with NSCLC and had primary tumor (2 $\mathrm{cm}$ or less) who underwent sublobar resection $(n=154)$ and lobectomy ( $n=84$ ) were examined. The results were not favor of sublobar resection and lobectomy was associated with longer survival and recurrence-free survival and less local recurrence. However, similar survival results were observed when compared lobectomy patients and 45 sublobar resection patients who were sampled with mediastinal lymph node during surgery [11].

In terms of the effect of International Association for the Study of Lung Cancer (IASLC)/American Thoracic Society (ATS)/ European Respiratory Society (ERS) adenocarcinoma classification on sublobar resections; if selected patients are offered sublobar resection, it is possible to reach cancer treatment with efficacy equivalent to lobectomy. The selection criteria are that the tumor is smaller than $2 \mathrm{~cm}$, the possibility to reach a resection limit of more than $1 \mathrm{~cm}$, and segmentectomy may be applied instead of wedge resection. The datas can be easily obtained from the standard CT used for lung cancer imaging. The datas that seen in CT to perform a successful sublobar resection are that the lesion is in the outer one third of the lung parenchyma, the lesion is smaller than $2 \mathrm{~cm}$ and there is no evidence of endobronchial involvement $[12,13]$. 
When these conditions are not provided, trying sublobar resections increases the risks as insufficient resection margins, separation in the stapler line and bending the remaining lung parenchyma after using staples. Another main factor in determining the sublobar resection patients is that the patient is not appropriate for lobectomy. Full lung function test is necessary, including spirometer, arterial blood gas analysis, and even determination of carbon monoxide diffusion capacity.

\section{Robotic segmentectomy}

In lung segment resections; segmentectomy has an equivalent morbidity, recurrence and survival rate compared to lobectomy, in patients with stage I lung cancer, tumors smaller than $2 \mathrm{~cm}$ and within the segmental anatomical limits. Segmentectomy also contributes more to preserving lung function and exercise capacity than lobectomy. In tumor resection; especially in patients with advanced age, insufficient performance or insufficient cardiopulmonary reserve, $2 \mathrm{~cm}$ in diameter and acceptable segmental margins may be procured [14].

Video-assisted thoracic surgery (VATS) is a minimally invasive approach for lung cancer surgery with several advantages according to open thoracotomy, it also includes some limitations such as rigid instruments and insufficient view [15]. Robot assisted thoracic surgery (RATS) technology is an evolution of manual video thoracoscopy that retain the advantages of low invasiveness. More intuitive movements, more flexibility and high definition, three-dimensional view are advantages of robotic approach. Different studies show that robotic lobectomy and robotic segmentectomy are feasible and safe with long-term results similar to open/ VATS approaches. Indications for performing robotic lung resections may be wider than traditional videothoracoscopic approach and may include patients with local advanced disease or requiring anatomical segmentectomy after chemotherapy [16].

In the study of Toker, et al., it was reported that 21 patients who underwent robotic lung anatomic segmentectomy with robot-assisted thoracoscopic surgery were reviewed, 15 patients (75\%) were operated on for malignant lung diseases, conversion to open surgery was not necessary. Postoperative complications occurred in 4 patients, mean duration of chest tube drainage was $3 \pm 2.1$ days, mean postoperative hospital stay was $4 \pm 1.4$ days. Robot-assisted thoracoscopic segmentectomy for malignant and benign lesions appears to be practical, safe, and associated with few complications and short postoperative hospitalization and lymph node removal also appears oncologically acceptable for early lung cancer patients were reported [17]. In the study of Wei, et al. it was stated that 100 patients underwent robotic segmentectomy, 7 patients were converted to lobectomy. 5 pneumothorax, 7 atrial fibrillation, 1 coagulopathy were observed as postoperative minor complications. Mean hospital stay was 2 days, 60 and 90 days mortality was 0 [18]. In another study in the literature, Pardolesi, et al. stated that 17 patients underwent a robot-assisted lung anatomic segmentectomy, there were no major intraoperative complications and conversion to open procedure was not required. Postoperative morbidity with pneumonia in 1 case and prolonged air leaks in 2 patients were seen. Median postoperative stay was 5 days, and postoperative mortality was $0 \%$. Due to these results, robotic anatomic lung segmentectomy as feasible and safe procedure were reported [19]. In the study of Kagimoto, et al. it was reported that 20 patients underwent robotic anatomical segmentectomy and robotic anatomical segmentectomy for early stage non-small cell lung cancer seems safe and feasible [20].

Considering studies analyzing results of robotic method with video-assisted thoracoscopic surgery method which is used almost as a standard in early stage cancers, Demir, et al. stated that 99 patients underwent RATS $(n=34)$ and VATS $(n=65)$ pulmonary segmentectomies, morbidity rates were $24 \%$ and $23 \%$ and mortality rates were $0 \%$ and $1.5 \%$ for RATS and VATS, respectively. The mean console time for RATS was longer than the mean operation time for VATS, the duration of postoperative stay for RATS was 4.65 days and for VATS was 6.16. Both RATS and VATS pulmonary segmentectomy operations were performed with similar morbidity and mortality rates, Although the duration of operation was longer in RATS when compared with an established VATS programme, there was a tendency towards a shorter postoperative stay were reported [21]. In the study of Zhou, et al. it was stated that 130 patients with resected stage IA non-small cell lung cancer underwent RATS $(n=50)$ and VATS $(n=80)$ pulmonary segmentectomy. Operation time, intensive care unit stay, hospital stay, and blood loss were reduced in the RATS group compared to the VATS group. The number of totally dissected lymph nodes and postoperative complications were similar between two groups, there was no operative mortality and there was no significant difference in the 5-year recurrence-free survival between the RATS and VATS groups (100\% vs. 93.75\%) were reported [22]. In the study of Liang, et al. it was stated that 7438 patients were included in the analysis, roboticassisted lobectomy/segmentectomy (RAL/S) was performed on 3239 patients, video-assisted lobectomy/segmentectomy (VAL/S) was performed on 4199 patients. This meta-analysis confirmed that RAL/S as a feasible and safe alternative to $\mathrm{VAL} / \mathrm{S}$ for resection of lung cancer was indicated [23]. In the study of Zhang, et al. it was reported that 774 patients underwent $($ RATS $=298$, VATS $=476)$ minimally invasive segmentectomy for early-stage NSCLC. 3 conversions to thoracotomy occurred in the VATS group, and 1 in the robotic group. There was no significant difference in rates of overall complications (17.9 vs. 14.8\%), and length of stay (4 days vs. 4 days). It was stated that segmentectomy with 
robotic and VATS was safe and feasible for early-stage NSCLC treatment and robotic approach led to better N1 lymph node dissection [24]. In the study of Xie, et al. it was stated that 166 patients were included in the analysis, 81 patients underwent RATS segmentectomy and 85 underwent VATS segmentectomy. The number of lymph nodes dissected in RATS group was more than in VATS group, the incidence of some postoperative complications such as pro-longed air leak, atrial fibrillation was not significant different between the two approaches were reported [25].

In terms of oncological results, in the study of Nguyen D. et al., it was stated that 71 patients had clinical stage I NSCLC underwent R0 resection. Median hospitalization was 4 days, complication rate was $29 \%$. Tere were no complications and no patient died within 90 days. Mean follow-up was 54 months, the overall 5-year survival was $43 \%$, lung cancer-specific 5 -year survival was $55 \%$ and the 5-year lung cancer-specific survival for pathological stage I disease was $73 \%$. Local or mediastinal recurrence occurred in 4 patients, pathological upstaging or recurrence resulted as $0 \%$. Robotic anatomical segmentectomy with mediastinal nodal dissection was a safe and feasible procedure and accurate preoperative clinical staging was of critical importance for long-term survival were reported [26]. In the study of Cerfolio, et al. it was stated that 93 patients underwent anatomic robotic segmentectomy. There were no conversions to thoracotomy, the median length of stay was 3 days and major morbidity occurred in 2 patients, there were no 30- or 90-day mortalities. Of the 79 patients with lung cancer, the median follow-up was 30 months, 3 patients had recurrence in the operated lobe and overall survival was $95 \%$ at 30 months. As a result, it was predicated robotic anatomic segmentectomy was safe and effective method [27].

\section{Conclusion}

Retrospective studies show that robot-assisted thoracic surgery is feasible and safe in non-small cell lung cancer treatment. Limited long-term results show oncological results of robotic approach similar to open and VATS approaches. Robotic approach facilitates surgery with more intuitive movements, greater flexibility and high definition, threedimensional vision. However, high cost and lack of touch sense are main disadvantages of robotic surgery. New studies are needed to assess quality of life, morbidity, oncological results and cost effectiveness. However, considering development of technology and increasing number of surgical robot brands in the near future, it is predicted that disadvantages of robotic surgery will be minimized in the near future. In the light of this information, with appropriate patient selection for segmentectomy in the treatment of lung cancer, robotic segmentectomy is considered to be safe and feasible based on current literature results.

\section{Acknowledgment}

Anil Gokce would like to thank Prof. Robert J. Cerfolio for the chance to work together about robotic thoracic surgery in New York University Langone Health Center and the contributions to this article.

\section{References}

1. Kalaycı G, Dilege S. Lung Resections. In: Yuksel M, Kalaycı (eds). Thoracic Surgery, 1stEdition. Istanbul:Bilmedya Group;2001;133-142. PubMed: https://www.ncbi.nlm.nih.gov/pmc/articles/PMC5009068/

2. Jensik RJ, Faber LP, Milloy FJ, Monson DO. Segmental resection for lung cancer- a fifteen-year experience. J Thorac Cardiovasc Surg. 1973; 66: 563-572.

PubMed: https://pubmed.ncbi.nlm.nih.gov/4356889/

3. Read RC, Yoder G, Schaeffer RC. Survival after conservative resection for T1 N0 M0 non-small cell lung cancer. Ann Thorac Surg. 1990; 49: 391-400.

PubMed: https://pubmed.ncbi.nlm.nih.gov/2155592/

4. Ginsberg RJ, Rubinstein LV. Randomized trial of lobectomy versus limited resection for T1 N0 non-small cell lung cancer. Lung Cancer Study Group. Ann Thorac Surg. 1995; 60: 615-623.

PubMed: https://pubmed.ncbi.nlm.nih.gov/7677489/

5. Nakamura H, Kawasaki N, Taguchi M, Kabasawa K. Survival following lobectomy vs limited resection for stage I lung cancer: a meta-analysis. Br J Cancer. 2005; 92: 1033-1037.

PubMed: https://www.ncbi.nlm.nih.gov/pmc/articles/PMC2361939/

6. De Zoysa MK, Hamed D, Routledge T, Scarci M. Is limited pulmonary resection equivalent to lobectomy for surgical management of stage I non-small-cell lung cancer? Interact Cardio Vasc Thorac Surg. 2012; 14: 816-820.

PubMed: https://pubmed.ncbi.nlm.nih.gov/22374287/

7. Harada H, Okada M, Sakamoto T, Matsuoka H, Tsubota N Functional advantage after radical segmentectomy versus lobectomy for lung cancer. Ann Thorac Surg. 2005; 80: 2041-2045. PubMed: https://pubmed.ncbi.nlm.nih.gov/16305841/

8. Kilic A, Schuchert MJ, Pettiford BL, Pennathur A, Landreneau JR, et al. Anatomic segmentectomy for stage I non-small cell lung cancer in the elderly. Ann Thorac Surg. 2009; 87: 1662-1668. PubMed: https://pubmed.ncbi.nlm.nih.gov/19463574/

9. Donahue JM, Morse CR, Wigle DA, Allen MS, Nichols FC, et al. Oncologic efficacy of anatomic segmentectomy in stage IA lung cancer patients with T1a tumors. Ann Thorac Surg. 2012; 93: 381-388. PubMed: https://pubmed.ncbi.nlm.nih.gov/22209375/

10. Kates M, Swanson S, Wisnivesky JP. Survival following lobectomy and limited resection for the treatment of stage I non-small cell lung cancer $\leq 1 \mathrm{~cm}$ in size: a review of SEER data. Chest. 2011; 139: 491-496. PubMed: https://pubmed.ncbi.nlm.nih.gov/20576736/

11. Wolf AS, Richards WG, Jaklitsch MT, Gill R, Chirieac LR, et al Lobectomy versus sublobar resection for small $(2 \mathrm{~cm}$ or less) nonsmall cell lung cancers. Ann Thorac Surg. 2011; 92: 1819-1825. PubMed: https://pubmed.ncbi.nlm.nih.gov/22051277/

12. Travis WD, Brambilla E, Noguchi M, et al. International association for the study of lung cancer/American Thoracic Society/European Respiratory Society international multidisciplinary classification of lung adenocarcinoma. J Thorac Onco. 2011; 6: 244-285. PubMed: https://www.ncbi.nlm.nih.gov/pmc/articles/PMC4513953/

13. Pettiford BL, Landreneau RJ. Role of sublobar resection (segmentectomy and wedge resection) in the surgical management of non-small cell lung cancer. In: Thoracic Surgery (3rd Edition). Patterson GA, Cooper JD, Deslauriers J, Lerut AEMR, Luketich JD, Rice TW, Pearson FG (Eds). Elsevier, Philadelphia, USA, 2008: 869-878. 
14. Yang CF, D'Amico TA. Open, thoracoscopic and robotic segmentectomy for lung cancer. Ann Cardiothorac Surg. 2014; 3: 142-152.

PubMed: https://www.ncbi.nlm.nih.gov/pmc/articles/PMC3988303/

15. Sezen CB, Aker C. VATS Lobectomy, Segmentectomy, Pneumonectomy. Updates on Pulmonary Diseases. 2020; 8: 37-42.

16. Veronesi G. Robotic lobectomy and segmentectomy for lung cancer: results and operating technique. J Thorac Dis. 2015; 7: 122-130. PubMed: https://www.ncbi.nlm.nih.gov/pmc/articles/PMC4419035/

17. Toker A, Ayalp K, Uyumaz E, Kaba E, Demirhan O, Erus S. Robotic lung segmentectomy for malignant and benign lesions. J Thorac Dis. 2014; 6: 937-942.

PubMed: https://www.ncbi.nlm.nih.gov/pmc/articles/PMC4120181/

18. Wei B, Cerfolio R. Technique of robotic segmentectomy. J Vis Surg. 2017; 14; 3: 140.

PubMed: https://www.ncbi.nlm.nih.gov/pmc/articles/PMC5676209/

19. Pardolesi A, Park B, Petrella F, Borri A, Gasparri R, et al. Robotic anatomic segmentectomy of the lung: technical aspects and initial results. Ann Thorac Surg. 2012; 94: 929-934.

20. Kagimoto A, Tsutani $Y$, Izaki $Y$, Handa $Y$, Mimae T, Miyata $Y$, Okada $M$. Initial experience of robotic anatomical segmentectomy for nonsmall cell lung cancer. Jpn J Clin Oncol. 2020; 50: 440-445. PubMed: https://pubmed.ncbi.nlm.nih.gov/32115630/

21. Demir A, Ayalp K, Ozkan B, Kaba E, Toker A. Robotic and videoassisted thoracic surgery lung segmentectomy for malignant and benign lesions. Interact Cardiovasc Thorac Surg. 2015; 20: 304-309. PubMed: https://pubmed.ncbi.nlm.nih.gov/25476458/
22. Zhou Q, Huang J, Pan F, Li J, Liu Y, et al. Operative outcomes and long-term survival of robotic-assisted segmentectomy for stage IA lung cancer compared with video-assisted thoracoscopic segmentectomy. Transl Lung Cancer Res. 2020; 9: 306-315. PubMed: https://www.ncbi.nlm.nih.gov/pmc/articles/PMC7225141/

23. Liang $\mathrm{H}$, Liang $\mathrm{W}$, Zhao L, Chen D, Zhang J, et al. Robotic versus video-assisted lobectomy/segmentectomy for lung cancer: a metaanalysis. Ann Surg. 2018; 268: 254-259.

PubMed: https://pubmed.ncbi.nlm.nih.gov/28628562/

24. Zhang $Y$, Chen $C$, Hu J, Han Y, Huang M, et al. Early outcomes of robotic versus thoracoscopic segmentectomy for early-stage lung cancer: a multi-institutional propensity score-matched analysis. J Thorac Cardiovasc Surg. 2020; S0022-5223(20)30226-9. PubMed: https://pubmed.ncbi.nlm.nih.gov/32113718/

25. Xie B, Sui T, Qin Y, Miao S, Jiao W. Comparison of short-term outcomes of lung segmentectomy by robotic-assisted and video-assisted thoracoscopic surgery. Zhongguo Fei Ai Za Zhi. 2019; 22: 767-771. PubMed: https://pubmed.ncbi.nlm.nih.gov/31874672/

26. Nguyen D, Gharagozloo F, Tempesta B, Meyer M, Gruessner A. Longterm results of robotic anatomical segmentectomy for early-stage nonsmall-cell lung cancer. Eur J Cardiothorac Surg. 2019; 55: 427-433. PubMed: https://pubmed.ncbi.nlm.nih.gov/30325403/

27. Cerfolio RJ, Watson C, Minnich DJ, Calloway S, Wei B. One hundred planned robotic segmentectomies: early results, technical details, and preferred port placement. Ann Thorac Surg. 2016; 101: 1089-1096. PubMed: https://pubmed.ncbi.nlm.nih.gov/26846343/ 